\title{
The Evolving Landscape of Primary Immunodeficiencies
}

\author{
Andrew R. Gennery ${ }^{1}$
}

Received: 15 March 2016 / Accepted: 16 March 2016/Published online: 23 March 2016

(C) Springer Science+Business Media New York 2016

To the Editor,

The landscape of primary immunodeficiencies continues to evolve at a gathering pace. Since the discovery of monogenic causes of primary immunodeficiency (PID), we have experienced many nuances but also seismic changes in our understanding of the manifestation of genetically inherited PID. We should no longer be surprised that defects in single genes can manifest in a variety of clinical manifestations, perhaps best typified by defects in recombination activating genes 1 and 2 (RAG1/2) [1]. First described as causing T-B-NK+ severe combined immunodeficiency (SCID), we learned that hypereosinophilia with immunodeficiency (Omenn syndrome) was caused, not only by hypomorphic mutations in the same genes, but in some families, the same mutation could manifest as these two extreme phenotypes in different individuals, emphasizing the co-influence of environmental factors on a genetic background in phenotypic evolution. Milder Omenn phenotypes, so called 'leaky' or atypical SCID, also due to $R A G 1 / 2$ mutations were described, but RAGassociated disease seemed to be firmly placed in the combined-immunodeficiency (CID) category of PID, even with the description of granulomatous lesions associated with $\mathrm{CID}$, or cytomegalovirus infection and an expansion of T-cell receptor $\gamma \delta$ T-lymphocytes. However, common variable immunodeficiency, agammglobulinaemia, adult-onset specific antibody deficiency, selective IgA deficiency and autoimmune cytopenias are now all recognized as bona fide manifestations of $R A G$ deficiency.

Andrew R. Gennery

a.r.gennery@ncl.ac.uk

1 Primary Immunodeficiency Group, Institute of Cellular Medicine, Newcastle University, Newcastle upon Tyne, UK
If $R A G$ deficiency has a promiscuous presentation, certain PIDs present with a stable phenotype. Examples include Wiskott Aldrich syndrome, classically presenting with a triad of eczema, microthrombocytopenia and immunodeficiency, or chronic granulomatous disease, characterized by recurrent bacterial and fungal infections, and organ inflammation, particularly of the respiratory and gastrointestinal tract. Whilst the specific environment may modify timing of presentation and manifestation of the specific infectious agent, the broad phenotype seems stable through time and geography, as far as we can ascertain at present.

A few genes have variable penetrance in particular kindreds, or function as disease modifiers. Heterozygous mutations in FAS are a cause of autoimmune lymphoproliferative syndrome, but penetrance is incomplete, with affected and non-affected family members in the same kindred [2]. Heterozygous mutations in $T A C I$ are associated with IgA deficiency and with common variable immunodeficiency. However, it seems increasingly likely that mutations in $T A C I$ act as modifiers and co-participants of disease, rather than as the primary cause [3].

The genetic and biological environment in which they present significantly modifies clinical manifestations of disease. Severe disease-causing mutations in genes, which predispose to infection with one or few micro-organisms, e.g. UNC93B1, mutations in which lead to herpes simplex encephalitis, only manifest upon exposure to the specific infectious agent. Affected patients may remain well for many years, or never manifest disease, depending when, if ever, exposure to herpes simplex occurs.

So in many ways, the report by Felgentreff et al. [4], in this issue of the Journal of Clinical Immunology, pages 341-353, should come as no surprise. A family is described, with the index case manifesting with recurrent infection, and hypogammaglobulinemia, associated with short stature and 
microcephaly. The phenotype is classic of DNA double strand breakage repair defects, and sequencing of LIG4 revealed two compound heterozygous mutations, each inherited from a parent. One mutation affecting the catalytic domain has previously been reported in patients with moderately severe disease. The other mutation affects the enzymatic domain, has not been reported previously but is predicted to have a deleterious effect. Detailed immunological and biochemical analysis demonstrated B-lymphocytopenia and defective class switching in B-lymphocytes, as well as reduced naïve $\mathrm{CD}^{+}$and $\mathrm{CD} 8^{+} \mathrm{T}$ lymphocytes and reduced mitogen-induced T-lymphocyte stimulation. CDR3 regions from T- and B-lymphocyte receptors were shorter than in controls. There was an increased use of microhomology-mediated end-joining of TRB and $\operatorname{IgH}$ sequences, and fibroblasts demonstrated increased radiosensitivity. Peripheral blood mononuclear cells showed an increased DNA damage following exposure to ionizing radiation and delayed DNA repair kinetics.

These results and clinical findings are uncontroversial. More surprisingly given the importance of DNA repair enzymes in lymphocyte receptor development, and in developing neurons, was the finding that two siblings of the patient carry the same mutations, but clinically are only very mildly affected or asymptomatic. However, laboratory investigations revealed variable results, with mild T-lymphocytopenia in one, but profound reduction in B-lymphocyte numbers in all genetically affected individuals, although in one asymptomatic individual the percentage of switched and unswitched memory B-lymphocytes was normal. Similar to the index case, cells from both asymptomatic $L I G 4$-mutated individuals displayed increased sensitivity to ionizing radiation with delayed repair kinetics, although less severe than the patient. Abnormalities in telomere length, CDR3 length, and increase in the use of micro-homology was also variably described.

In some ways, what makes these findings so surprising is not that LIG4-mutated individuals may be normal, for the first patient described with LIG4 deficiency showed no abnormalities until treated with chemotherapy and radiotherapy. Instead, it is the intra-familiar variation in clinical phenotype for a gene that appears so critical for normal development of the central nervous system and adaptive immunity, and which demonstrated biochemical effects in all mutated individuals without parallel clinical manifestation. That different mutations may confer diverse phenotypes is acceptable, but the same mutation causing similar disrupted function, with different clinical phenotypes, and even conferring laboratoryascertained abnormalities that do not seemingly impact 'real-life' function, is difficult to comprehend. Presumably these individuals would have shared a broadly similar external environment, and genetic background, and so it is challenging to immediately explain the different presentations in the kindred described.
In patients with mutations in $X R C C 4$, similar biochemical abnormalities to those documented here are described, although no patients described so far display an overt immunological phenotype [5]. Familial hemophagocytic lymphohistiocytosis (FHL) classically presents with prolonged fevers, cytopenias, hepatosplenomegaly, and signs of immune activation, usually in early childhood. However, hypomorphic mutations in FHL-associated genes confer a more indolent, late-onset disease phenotype, often appearing in adulthood.

The first described PIDs have identified severe mutations in genes that contribute a significant impact on the development of immunity. Slowly we have appreciated that less severe mutations modify disease presentation, and defects in genes that are less important for global immune development only manifest in narrow circumstances. Mutations that preserve some function present with less severe phenotypes and often later in life, and mutations in some genes appear to act as co-modifiers with other defects in order that disease may present. Given the complexity of our immune system and our genetic make-up, it would not be surprising if defects in genes hitherto considered critical for immune development or regulation were also modified by other genetic, epigenetic and environmental factors. In patients with severe mutations or mutations in genes with critical function, the effect of such modifiers is small, and lost in the impact of the primary mutation. In less severe phenotypes, the effect of such modifiers becomes more pronounced. Sherlock Holmes observed that "the world is full of obvious things which nobody by any chance ever observes." Careful descriptive examination of patients with PID, and of family members will help as we integrate data from new generation sequencing with clinical and laboratory information - and appreciate the biological and genetic complexity of even severe PID.

\section{References}

1. Walter JE, Rosen LB, Csomos K, et al. Broad-spectrum antibodies against self-antigens and cytokines in RAG deficiency. J Clin Invest. 2015;125:4135-48.

2. Price S, Shaw PA, Seitz A, et al. Natural history of autoimmune lymphoproliferative syndrome associated with FAS gene mutations. Blood. 2014;123:1989-99.

3. Salzer U, Bacchelli C, Buckridge S, et al. Relevance of biallelic versus monoallelic TNFRSF13B mutations in distinguishing disease-causing from risk-increasing TNFRSF13B variants in antibody deficiency syndromes. Blood. 2009;113:1967-76.

4. Felgentreff K, Baxi SN, Lee YN, et al. Ligase-4 deficiency causes distinctive immune abnormalities in asymptomatic individuals. $\mathrm{J}$ Clin Immunol. 2016;36:341-353.

5. Guo C, Nakazawa Y, Woodbine L, et al. XRCC4 deficiency in human subjects causes a marked neurological phenotype but no overt immunodeficiency. J Allergy Clin Immunol. 2015;136:1007-17. 